\title{
Polymorphisms of the TAP1 and TAP2 transporter genes in Japanese SLE
}

\author{
F Takeuchi, K Nakano, H Nabeta, G H Hong, S Kuwata, K Ito
}

\begin{abstract}
Objective-To determine how polymorphism of transporter associated with antigen processing 1 and 2 (TAP1 and 2) alleles contributed to the pathogenesis of systemic lupus erythematosus (SLE) in Japanese patients.

Methods-TAP1 and TAP2 typing was carried out in 52 Japanese patients with SLE and 95 normal subjects by the PCR-RFLP (restriction fragment length polymorphism) method. HLA-DR typing and HLA-DRB1*15 genotyping were carried out by the PCR method and PCRSSCP (single stranded DNA conformation polymorphism) method, respectively. Results-No particular TAP 1 allele was associated with Japanese SLE or with immunological subgroup of SLE. TAP2H showed a tendency towards increased frequency in SLE $(5.8 \% v 0 \%$ in control), but the corrected $P$ value was not significant. No other particular association of TAP2 allele was observed. Furthermore, these was no evidence for linkage disequilibrium between any TAP1/TAP2 alleles and HLA-DRB1 ${ }^{1501}$ - which is reported to be weakly but significantly association with Japanese SLE-in either the normal control or the SLE patient group.

Conclusions-Neither the TAP1 nor the TAP2 gene appears to determine disease susceptibility to SLE in Japanese, and these results are in keeping with those reported in Caucasian SLE patients.
\end{abstract}

(Ann Rheum Dis 1996;55:924-926)

Faculty of Medicine, University of Tokyo, Tokyo, Japan:

Department of

Medicine and Physical

Therapy

F Takeuch

K Nakano

H Nabeta

G H Hong

$\mathrm{K}$ Ito

Department of

Transfusion Medicine and Immunology

S Kuwata

Correspondence to:

Fujio Takeuchi MD

Department of Medicine and Physical Therapy, Faculty of

Medicine, University of

Tokyo, 7-3-1 Hongo,

Bunkyo-ku, Tokyo 113

Japan.

Accepted for publication 4 September 1996 including the presence of various autoan ies. The role of autoantibodies in the pathogenesis of SLE is still unclear. The contribution of genetic factors in the pathogenesis has been demonstrated by twin studies and family studies. Genetic predisposition was also suggested by associations with SLE of genes within the major histocompatibility complex (MHC), although these results are still controversial among different ethnic groups. In Japanese and Koreans, a weak but significant association between HLADRB $1^{\star} 1501$ and SLE patients has been reported. $^{12}$ Associations with complement C4AQ0 have been observed in both Japanese and Caucasian SLE patients. Several studies have suggested that HLA-DQ genes determined susceptibility to SLE. ${ }^{34}$
Transporter associated with antigen processing genes (TAP1 and TAP2), located between DQB1 and DRB1 loci, are included among the several factors involved in antigen processing which have recently been found to be encoded in the MHC class II region. TAP is homologous to the ATP binding cassette transporter superfamily, and polymorphisms of both TAP1 and TAP2 have been reported. ${ }^{5}$ In the rat, polymorphisms of TAP genes contribute to the spectrum of peptides bound to MHC class I molecule. ${ }^{6}$ Though TAP essentially participates in the delivery of peptides to the HLA class I molecule, ${ }^{7}$ the contribution of TAP to HIA class II restricted endogenous processing has also been reported. ${ }^{8}$ TAP gene is an interesting candidate as a disease susceptible gene or a genetic marker for SLE. After studying the associations between TAP2 gene and SLE in Caucasians, Davis et al reported that no particular TAP2 dimorphism or allele was involved in SLE. ${ }^{9}$ Savage et al reported no association between TAP1 and TAP2 alleles and Chinese SLE either. ${ }^{10}$

In this report, we studied polymorphism of TAP1 and TAP2 genes in 52 Japanese SLE patients to clarify the contribution of TAP genes to SLE using the PCR-RFLP (polymerase chain reaction-restriction fragment length polymorphism) method. The associations between TAP and HLA-DRB1*1501 gene are also discussed.

\section{Methods}

PATIENTS

Genomic DNA was obtained from the peripheral white blood cells of 52 randomly selected unrelated Japanese SLE patients [aged 41.5 (SD 12.0) years; 49 women and three men], diagnosed according to the criteria of the American Rheumatism Association. ${ }^{11}$ The control population comprised 95 unrelated healthy volunteers.
GENOTYPING OF TAP1 AND TAP2 GENES

TAP1 and TAP2 genotyping was performed by the PCR-RFLP method using the specific primers described previously. ${ }^{12}{ }^{13}$ In brief, two dimorphic sites of TAP1-codons 333 and 637-were digested with Sau3AI and AccI, respectively, and four dimorphic sites of TAP2 - codons 379, 565, 665, and 687-were digested using AccII, RsaI, MspI, and BfaI, respectively, after specific amplification. Four possible TAP1 alleles (TAP1A-D) and eight possible TAP2 alleles (TAP2A-H) were then determined and assigned according to the nomenclature proposed by Powis et $a l^{5}$ as described previously. ${ }^{1213}$ 
Table 1 Phenotype frequencies (\%) of TAP1 alleles in SLE and control populations

\begin{tabular}{llll}
\hline Allele & $A$ & $B$ & $C$ \\
\hline SLE (n=52) & 100 & 26.9 & 1.9 \\
dsDNA (n=25) & 100 & 28.0 & 0 \\
SS-A (n=18) & 100 & 16.7 & 0 \\
SS-B (n=2) & 100 & 50.0 & 0 \\
RNP (n=17) & 100 & 23.5 & 0 \\
Sm (n=5) & 100 & 40.0 & 0 \\
Control (n=95) & 96.8 & 26.3 & 1.1 \\
\hline
\end{tabular}

dsDNA, circulating antibodies to dsDNA; SS-A, circulating to SS-B; RN circulating antibodies to UI-RNP; Sm, circulating antibodies to $\mathrm{Sm}$.

\section{HLA-DR TYPING}

HLA-DR typing was performed on the second exon of HLA-DRB1 by the PCR method using the specific primers described previously. ${ }^{14}$

GENOTYPING OF DR 15

HLA-DR15 genes were genotyped by the PCR-SSCP (single stranded DNA conformation polymorphism) method using the specific primers and electrophoresis condition described previously. ${ }^{14}$

DETECTION OF AUTOANTIBODIES

Anti SS-A, SS-B, Ul-RNP, and Sm antibodies (a-SS-A, a-SS-B, a-RNP, a-Sm, respectively) were detected by the double immunodiffusion method using ENA-1 and ENA-2 test kit (MBL Co, Nagoya, Japan). Anti double stranded DNA antibody (a-dsDNA) was detected and quantified using an enzyme linked immunosorbent assay (ELISA) anti dsDNA kit (Japan DPC Co, Tokyo, Japan). These methods are standard in the Central Laboratory Service of Tokyo University Hospital. $^{14}$

\section{STATISTICS}

The $\chi^{2}$ test (with Yates correction) was used for comparison. When a value below 5 was contained in the $2 \times 2$ table, Fisher's exact test was used. Relative risk (RR) was calculated by standard Woolf's formula or, when " 0 " was included by Haldane's modified formula. Probability value (P) was corrected occasionally for the number of comparisons made [corrected probability $(\mathrm{Pc})]$. In this report, the $\mathbf{P}$ value was used primarily for comparison and discussion in order to avoid missing any clinically important associations, although Pc was used occasionally for statistical decisions.

\section{Results}

The phenotype frequencies of TAP1 in patients with SLE and controls are shown in table 1. No TAP1 allele was found to be

Table 2 Phenotype frequencies (\%) of TAP2 alleles in SLE and control populations

\begin{tabular}{llllllll}
\hline Allele & $A$ & $B$ & $C$ & $D$ & $E$ & $F$ & $G$ \\
\hline SLE (n=52) & 67.3 & 65.4 & 26.9 & 3.8 & 9.6 & 0 & $5.8^{\mathrm{a}}$ \\
dsDNA (n=25) & 76.0 & 64.0 & 28.0 & 4.0 & 12.0 & 0 & 0 \\
SS-A (n=18) & 83.3 & 66.7 & 22.2 & 5.6 & 5.6 & 0 & 0 \\
SS-B (n=2) & 100.0 & 50.0 & 0 & 50.0 & 0 & 0 & 0 \\
RNP (n=17) & 58.8 & 70.6 & 23.5 & 0 & 11.8 & 0 & 11.8 \\
Sm (n=5) & 40.0 & 100.0 & 0 & 0 & 40.0 & 0 & 0 \\
Control (n=95) & 65.3 & 60.0 & 23.2 & 2.1 & 18.9 & 1.1 & 0
\end{tabular}

dsDNA, circulating antibodies to dsDNA; SS-A, circulating antibodies to SS-A; SS-B, circulating antibodies to SS-B; RNP, circulating antibodies to UI-RNP; Sm, circulating antibodies to $\mathrm{Sm}$. a: $\mathrm{P}<0.05, \mathrm{Pc}=\mathrm{NS}, \mathrm{RR}=13.5$. significantly associated with SLE. TAP1 phenotype frequencies were also determined in various immunological subgroups of SLE. TAP1B frequency was slightly decreased in patients with circulating anti SS-A antibody $(P$ = NS). No significant associations of TAP1 alleles were observed in each immunological subgroup with autoantibody circulating to each autoantigen.

Table 2 shows TAP2 phenotype frequencies in SLE and controls. In SLE patients, the phenotype frequency of TAP2H was increased in comparison with control $(5.8 \% v 0 \%, \mathrm{P}<$ $0.05)$, but the increase was not statistically significant when the $\mathrm{P}$ value was corrected $(\mathrm{Pc}=$ NS). Phenotype frequency of TAP2E was slightly decreased in SLE in comparison with control, but again the difference was not significant. No associations were observed between TAP2 alleles and overall SLE either. TAP2 phenotype frequencies were also determined in various immunological subgroups of SLE. Patients with a-RNP showed slight but not significant increase in the phenotype frequency of TAP2H $(11.8 \%, \mathrm{P}=\mathrm{NS})$. SLE patients with a-SS-A showed a slightly high prevalence of TAP2A and low prevalence of TAP2E, but these differences were not significant. The frequency of TAP2E was not significantly increased in patients with circulating a-Sm. No TAP2 alleles were significantly associated in any immunological subgroup with autoantibody circulating to each autoantigen studied.

In control, associations of TAP1 and TAP2 alleles with $\mathrm{DRB} 1^{\star} 1501$, which is reported to be associated with Japanese and Korean SLE, ${ }^{12}$ were estimated. No significant association was observed between any TAP1 or TAP2 allele and DRB1 ${ }^{\star} 1501$ in either control or SLE.

\section{Discussion}

Because of their roles in the processing of antigen, TAP1 and TAP2 genes are potentially important in immune responses. ${ }^{6-8}$ Associations of TAP1 and TAP2 alleles have been studied in many diseases, including various autoimmune diseases, based on the hypothesis that TAP genes may represent candidate susceptibility genes or serve as useful markers for MHC linked diseases. ${ }^{9}$

In this experiment, a slightly increased frequency of TAP2H was observed in SLE, but the increase was not significant statistically $(P$ $<0.05, \mathrm{Pc}=\mathrm{NS}$ ). Additionally, the prevalence of TAP2H in SLE was too small $(5.8 \%)$ to consider it a disease susceptible factor. Our study ultimately indicates that no TAP1 and TAP2 alleles are associated with Japanese SLE. Neither was there any significant association of any TAP1 or TAP2 allele in any of the immunological subgroups of Japanese SLE studied. These results strongly suggest that TAP 1 and TAP2 are not genetically involved in the pathogenesis of Japanese SLE.

Davis et al indicated that TAP2 is not involved in determining genetic predisposition to SLE in Caucasians. ${ }^{9}$ Our results further supported their findings, adding to the report 
of Chinese SLE, ${ }^{10}$ though the genetic backgrounds of autoimmune diseases often differ between Japanese and Caucasians. In their report, no significant increases of TAP2C or TAP2D were observed in patients with a-SS-A or a-RNP. ${ }^{9}$ TAP2C was not statistically frequent in the Japanese a-SS-A subgroup we observed. TAP2D is a rare allele in Japanese and was not increased in Japanese patients with a-RNP. Several weak associations (not significant) observed in this study were not observed in their report. TAP2A, which was somewhat increased in Japanese SLE patients with a-SS-A, has been reported to be decreased in Caucasian SLE patients with a-SS-A. ${ }^{9}$ These findings in Japanese and Caucasian SLE patients ${ }^{9}$ strongly suggested that TAP2 (and TAP1) genes are not involved in the autoantibodies in the SLE studied.

A general association of TAP1 and TAP2 genes with HLA-DR in Japanese was reported previously. ${ }^{13}$ In this paper, the association of TAP alleles with HLA DRB ${ }^{\star} 1501$ was studied because a weak but significant association of HLADRB1*1501 had been observed in Japanese and Korean SLE patients. ${ }^{12}$ Although Singal et al ${ }^{15}$ additionally implicated TAP genes together with HLA-DR in rheumatoid arthritis, TAP genes did not contribute to the pathogenesis of Japanese SLE along with HLA DRB ${ }^{\star} 1501$. These findings essentially supported the previous observations by Davis et al. ${ }^{9}$ Davis reported a tendency towards increased frequency of TAP2D in SLE with a-RNP and the linkage disequilibrium between TAP2D and DR4 in control. ${ }^{9}$ Although the main genotypes of HLA-DRB $1{ }^{\star} 04$ differ between Japanese and Caucasians, TAP2D is a rare allele and is not associated with DR4 in Japanese controls. In the Chinese TAP2D is also a rare allele and is not associated with DR4 either. ${ }^{10}$

It had been reported that no particular TAP2 allele is associated with vasculitis and renal disease in SLE. ${ }^{9}$ In our study, associations between TAP alleles and vasculitis or renal disease are not shown since biopsies were not carried out in all SLE patients and accurate discrimination of vasculitis or renal disease was difficult clinically in several patients.

Our study strongly suggests that TAP1 and TAP2 genes do not determine disease susceptibility to Japanese SLE. As indicated in the Discussion of the previous report by Davis et $a l,{ }^{9}$ these results will prove useful in improving our understanding of the participation of TAP genes in the pathogenesis of SLE in a diverse range of ethnic groups.

This study was supported by the grants from the Ministry of Education, Science and Culture of Japan, the Ministry of Health and Welfare of Japan, and The Manabe Foundation. Health and Welfare of Japan, and The Manabe Foundation. fumi Yamada of Tokyo University, and Dr Kiyoaki Tanimoto of Saitama University.

1 Hong GH, Yamada H, Yu CL, Nakano K, Park KS, Kim $\mathrm{HY}$, et al. Association of MHC class II and III genes with SLE in Korean, Japanese and Chinese?.7th APLAR ConSLE in Korean, Japanese and Chin
gress of Rheumatology 1992:51.

2 Hong GH, Kim HY, Takeuchi F, Nakano K, Yamada $H$ Matsuta $\mathrm{K}$, et al. Association of complement $\mathrm{C} 4$ and HLA-DR alleles with systemic lupus erytematous in Korea. F Rheumatol 1994;21:442-7.

3 Arnett FC, Bias WB, Reveille JD. Genetic studies in Siogren's syndrome and systemic lupus erythematosus. $\mathcal{F}$ Autoimmunity 1989;2:403-13.

4 Davis EJ, Hillarby MC, Cooper RG, Hay EM, Green TR, Shah S, et al. HLA-DQ, DR and complement C4 variants in systemic lupus erythematosus. $\mathrm{Br} \mathcal{F}$ Rheumatol 1993 32:870-5.

5 Powis SH, Tonks S, Mockridge I, Kelly AP, Bodmer JG Trowsdale J. Alleles and haplotypes of the MHC-encoded ABC transporters TAP1 and TAP2. Immunogenetics 1993; 37:373-80.

6 Powis SJ, Deverson EV, Coadwell WJ, Ciruela A, Huskisson NS, Smith H, et al. Effect of polymorphism of an MHC-linked transporter on the peptides assembled in class I molecule. Nature 1992;357:211-5.

7 Braciale TJ, Brasiale VL. Antigen presentation: structural themes and functional variations. Immunol Today 1991 12:124-9.

8 Malnati MS, Marti M, LaVaute T, Jaraquemada D, Biddison W, DeMars R, et al. Processing pathways for presentation of cytosolic antigen to MHC class II presentation of cytosolic antigen to
restricted T cells. Nature 1992;357:702-4.

9 Davis EJ, Donn RP, Hillarby MC, Grennan DM, Ollier WER. Polymorphisms of the TAP2 transporter genes in systemic lupus erythematosus. Ann Rheum Dis 1994 53:61-3.

10 Savage DA, $\mathrm{Ng}$ SC, Howe HS, Ngai JLF, Darke C, Hui KM. HLA and TAP association in Chinese systemic lupus erythematosus patients. Tissue Antigens 1995;46:213-6.

11 Tan EM, Chohen AS, Fries JF, Masi AT, McShane DJ, Rothfield NF, et al. The 1982 revised criteria for the classification of systemic lupus erythematosus. Arthritis Rheum 1982;25:1271-7.

12 Kuwata S, Yanagisawa M, Saeki H, Nakagawa $H$, Etoh $T$, Tokunaga $\mathrm{K}$, et al. Lack of primary association between ransporter associated with antigen processing genes and ransporter associated with antigen processing genes and 96(suppl):1051-9.

13 Takeuchi F, Kuwata K, Nakano K, Nabeta H, Hong GH, Shibata Y, et al. Association of TAP1 and TAP2 with systemic sclerosis in Japanese. Clin Exp Rheumatol 1996 14:513-21.

14 Takeuchi F, Nakano K, Yamada H, Hong GH, Nabeta $H$, Yoshida A, et al. Association of HLA-DR with progressive systemic sclerosis in Japanese. $\mathcal{F}$ Rheumatol 1994;21:85763.

15 Singal DP, Ye M, Qiu X, D'Souza M. Polymorphism in the TAP2 gene and their association with rheumatoid arthritis. Clin Exp Rheumatol 1994;12:29-33. 